\title{
Perfil de cefaleias e proposta de ação em saúde
}

DOI: $10.5935 / 2447-8539.201900017$

\section{CAMILA FLÁVIO DE LIMA}

e-mail: cl.camilalima@outlook.com

\section{Resumo}

As cefaleias são manifestações de dor que acometem a região craniana, sendo uma dor frequente e de alta prevalência na população geral. A cefaleia pode ocorrer de forma isolada, fazer parte de um complexo sintomatológico ou secundária a uma doença instalada. Objetivo: Analisar o perfil de cefaleias em um grupo de indivíduos frequentadores como pacientes ou acompanhantes, em um ambulatório de clínica médica vinculado ao Curso de Medicina do Centro Universitário IMEPAC em Araguari - Minas Gerais (MG), objetivando uma proposta de intervenção em saúde para a população desta cidade. Metodologia: Estudo observacional, analítico, transversal, quantitativo com dados colhidos através de aplicação de questionário próprio em 100 indivíduos frequentadores como pacientes ou acompanhantes em um ambulatório universitário de clínica médica geral. Resultados: Entre os 100 entrevistados, apenas 87 foram incluídos, destes, 46 relataram ter apresentado cefaleia em alguma época de suas vidas ou que ainda apresentam cefaleia na atualidade. Destes 46 pacientes, 42 são do sexo feminino e 4 do sexo masculino. Destes 46 pacientes, 15 tiveram sua cefaleia classificada como migrânea sem aura, 19 como migrânea com aura, 11 como cefaleia tensional. Conclusão: Para que a população tenha acesso a assistência adequada, é necessária uma equipe multidisciplinar composta por Médicos, Nutricionistas, Psicólogos e Educadores Físicos aptos a desenvolverem ações de promoção da saúde que visam a adequação de hábitos de vida saudáveis e oferta de ações educativas, incentivando uma alimentação adequada e balanceada, bem como a prática de atividades

Palavras-chave: Cefaleia, Ambulatório, Prevalência, Questionário.

\section{Abstract}

Headaches are results of pain which affect the cranial region by being a constant and highly dominant pain in the general population. The headache symptom may occur in isolation as part of a complex symptomalogical or a result of a disease in the human body. The aim of this work is to analyze the types of headaches in a group of individual who are under care also the out patients at a medical clinic in partnership to the medical course at the IMEPAC in Araguari in the state of Minas Gerais. This analyses aims for a health intervention proposal for the population of this city. The used method is through an observational,analytical, cross sectional and quantitative study by collecting data by means of an application of a questionnaire to 100 patients and out patients at the university's general medical practice. The results among the 100 interviewees were that 13 had to be excluded due to the problems with the software used to submit the questionnaires. It is found that 46 out the remaining 87 interviewees reported that have had headaches at some time in their lives or still having headaches today. From these 46 patients, 42 are female and 4 male. Also 15 out of these 46 patients had headache classified as migraine without aura, 19 as migraine with aura, 11 as tensioned headache. So that the population may have access to proper care, a multidisciplinary team of physicians, nutritionists, psychologists and physical educators capable of developing health actions is important to promoting healthy living habits and offering educational actions such as encouraging a proper and balanced diet, the practice of physical and mental activities regularly as well as making the population aware of leaving the addictions and stressful behaviors behind.

Keywords: Headache. Medical Ambulatory. Prevalence. Questionnaire 


\section{INTRODUÇÃO}

A cefaleia é uma queixa universal e frequente na prática médica, sua extensa gama de subtipos faz com que todos os pacientes possam experimentá-la. Descrita por Randall (2001) como dor difusa em qualquer região da cabeça, que não está restrita a área atingida por um nervo específico. Sua manifestação pode ser desde uma dor branda e quase imperceptível até crises graves com risco de vida (PORTO, 2016).

Esses diversos subtipos são divididos em dois grupos principais: cefaleias primárias aquelas onde a própria queixa é em si a doença, inclui enxaqueca ou migrânea com ou sem aura, tensional frequente ou pouco frequente, em salvas, hemicraniana paroxística crônica e pós-traumática. E cefaleias secundárias, aquelas que são consequentes de algum quadro, estas dividem-se ainda em 3 grupos: intracraniana, consequente de acidente vascular encefálico, hemorragia subaracnóidea, hematoma subdural, hipertensão intracraniana e outras causas. Extracraniana, quando a dor decorre de situações como alterações da coluna cervical, disfunção temporomandibular, neuralgia do trigêmeo e outros. E por fim as de causas sistêmicas advindas de febre de qualquer etiologia, hipertensão arterial sistêmica (HAS), anemia, síndrome de abstinência, abuso de analgésicos e anti-inflamatórios e outros (PORTO, 2016).

A abordagem dos pacientes com cefaleia deve ser minuciosa, um exame clínico bem aplicado pode chegar a um diagnóstico conclusivo em até $90 \%$ dos casos (PORTO, 2016), a anamnese detalhada deve valorizar dados como: tipo, localização, irradiação, intensidade, evolução, duração e periodicidade da dor, sintomas associados, fatores de melhora ou piora, história familiar, estado emocional, tratamentos já utilizados e suas eficácias, uso de medicamentos e drogas ilícitas, doenças sistêmicas, agudas ou crônicas, febre, náuseas, vômitos, distúrbios visuais, tonturas, fotofobia, fonofobia, síncopes,

convulsões, emagrecimento, anemia e parestesias. (PORTO, 2016).

A Classificação Internacional de Cefaleias (CIC) de 2014 em sua terceira edição utiliza descrições que ajudam na diferenciação entre os subtipos da cefaleia. Este estudo usou como foco as causas primárias que a literatura referência como mais prevalentes.

Suas características clínicas são de acordo a CIC:

- migrânea sem aura (MSA): cefaleia de localização unilateral, caráter pulsátil, intensidade moderada ou forte, associada a náusea e/ou fotofobia e/ou fonofobia. Agravamento com atividades físicas de rotina. As crises duram de 4 a 72 horas.
- migrânea com aura (MCA): Localização unilateral, caráter pulsátil, intensidade moderada ou forte, associada a náusea e/ou fotofobia e/ou fonofobia. As crises duram de 4 a 72 horas. A aura é o complexo de sintomas totalmente reversíveis da fala e/ou linguagem, do tronco cerebral, motores, visuais, sensitivos e retinianos que acontece imediatamente antes ou no início da crise álgica.

- cefaleia tensional (CT): Localização bilateral, caráter em aperto ou pressão, de leve a moderada intensidade, durando de 30 minutos a 7 dias, não se agrava por atividade física rotineira, com ausência de náuseas ou vômitos e podendo estar presentes fotofobia e/ou fonofobia.

Por seu caráter limitante, frequentemente causando prejuízos profissionais e sociais, e pela grande oneração e demanda do sistema de saúde que causa, a cefaleia se mostra uma importante manifestação clínica a ser compreendida e confrontada. Entender suas principais causas e epidemiologia é vital para a tomada de ações preventivas que podem ser feitas ainda mesmo na atenção básica, portanto o estudo traz também uma proposta de saúde coletiva que visa disponibilizar nas Unidades Básicas de Saúde da Família (UBSF) informações quanto a prevenção e alívio de crises de cefaleia específicas para a população do município.

Frente ao exposto o objetivo deste estudo foi descrever o perfil de distribuição dos tipos mais prevalentes de cefaleias em um ambulatório universitário de clínica médica na cidade de Araguari - MG.

\section{METODOLOGIA}

A pesquisa aqui descrita configura-se como um estudo observacional, analítico, transversal, quantitativo com dados coletados através de entrevistas mediante aplicação de questionário próprio em um ambulatório universitário de clínica médica em Araguari - MG.

A amostra de conveniência foi instituída em pacientes ou acompanhantes de pacientes que frequentava esse ambiente, no período de agosto a novembro de 2019, totalizando 100 entrevistados, tendo-se descartado 13 sobrando uma amostra de 87 indivíduos dentre os quais 46 entrevistados relataram dores de cabeça.

As entrevistas e a aplicação dos questionários foram realizadas no espaço físico do ambulatório mediante a assinatura do termo de consentimento livre e esclarecido independentemente de sexo, etnia, religião, escolaridade e idade e que responderam adequadamente ao questionário.

Foram excluídos pacientes que tiveram sua cefaleia classificada como secundária, pacientes com déficit 
cognitivo e aqueles impossibilitados de assinar o termo de consentimento livre e esclarecido. O questionário compõe-se por 10 itens: número de crises, duração da dor, localização da dor, caráter da dor, intensidade da dor, fatores associados de piora e melhora, sinais e sintomas que acompanham a dor, duração de cada sinal ou sintoma, início da cefaleia e presença de alguma doença que justifique a dor, com critérios diagnósticos anexados ao questionário.

O estudo foi autorizado pelo Comitê de Ética em Pesquisa do Instituto Master de Ensino Presidente Antônio Carlos e justifica-se pela sua originalidade e seu aspecto relevante no mundo contemporâneo.

\section{Critérios diagnósticos de cefaleia}

Critérios diagnósticos de migrânea (enxaqueca) sem aura

A. Pelo menos cinco crises preenchendo os critérios de B a D.

B. Cefaleia durando de 4 a 72 horas (sem tratamento ou com tratamento ineficaz.

C. Cefaleia preenche ao menos duas das seguintes características:

- Localização unilateral.

- Caráter pulsátil.

- Intensidade moderada ou forte.

- Exacerbada por, ou levando o indivíduo a evitar atividades físicas. D. Durante a cefaleia, pelo menos um dos seguintes sintomas:

- Náusea e/ou vômitos.

- Fotofobia ou fonofobia. E. Não atribuída a outro transtorno.

Critérios diagnósticos para cefaleia migrânea (enxaqueca) com aura

A. Pelo menos duas crises que preenchem os critérios de B a D.

B. Aura consistindo em pelo menos um dos seguintes:

- Sintomas visuais completamente reversíveis, incluindo características positivas (p. ex., luzes tremulantes, manchas ou linhas) e/ou características negativas (p. ex., perda visual)

- Sintomas sensitivos completamente reversíveis, incluindo características positivas (p. ex. formigamento) e/ou características negativas (p. ex., adormecimento).
- Alteração da linguagem (afasia) completamente reversível.

C. Pelo menos dois dos seguintes:

- Sintomas visuais homônimos e/ou sintomas sensitivos unilaterais.

- Pelo menos um sintoma de aura desenvolve-se gradualmente em cinco minutos e/ou diferentes sintomas de aura ocorrem em sucessão de cinco minutos.

- Cada sintoma dura entre 5 e 60 minutos.

D. Cefaleia que preenche os critérios de B a D para migrânea sem aura começa durante a aura ou dentro de 60 minutos do início dela. E. Não atribuída a outro transtorno.

\section{Critérios diagnósticos para cefaleia tipo tensão}

A. Cefaleia tem pelo menos duas das seguintes características:

- Localização bilateral.

- Caráter em pressão/aperto (não pulsátil).

- Intensidade fraca a moderada.

- Não é agravada por atividades físicas rotineiras como caminhar ou subir escadas.

B. Ambos os seguintes:

- Ausência de náusea ou vômitos (anorexia pode ocorrer).

- Fotofobia ou fonofobia (apenas uma delas pode estar presente).

C. Não atribuída a outros transtornos.

\section{RESULTADOS}

Foram aplicados questionários direcionados para o melhor conhecimento sobre a cefaleia e seu acometimento em pacientes usuários do Sistema Único de Saúde. A coleta dos dados foi realizada no Centro Ambulatorial Dr. Romes Nader, em Araguari - MG vinculado ao Centro Universitário IMEPAC.

Foram entrevistados um total de 100 (cem) indivíduos escolhidos aleatoriamente entre os presentes neste local na condição de pacientes. Devido a problemas ocorridos durante a coleta do material com o software dos questionários 13 (treze) fichas de respostas foram parcialmente deletadas sendo, portanto, excluídas do 
estudo. A amostra restante foi de 87 indivíduos, 66 mulheres e 21 homens.

Destes 87 indivíduos, 46 relataram algum tipo de cefaleia, descritas como 19 migrâneas com aura $(41,3 \%)$ destes sendo 18 mulheres (94\%) e 1 homem (6\%), 15 migrâneas sem aura (32,6\%) destes sendo 14 mulheres $(93,3 \%)$ e 1 homem $(6,6 \%), 11$ tensionais $(23,9 \%)$ sendo 9 mulheres (81\%) e 2 homens (19\%) e 1 que não se encaixa em nenhuma das estudadas neste trabalho.

Quanto aos hábitos de vida, 21 entrevistados (24\%) informaram ser tabagistas, porém dentre os 21 tabagistas, 10 (47\%) relataram algum tipo de cefaleia. Em seguida, 36 entrevistados (41\%) informaram ser etilistas, onde 16 (34\%) relataram sofrer de cefaleia, 51 (58\%) entrevistados declararam-se sedentário e deles 27 (58\%) têm cefaleia. E 45 (51\%) declararam praticar alguma atividade física enquanto destes 19 (41\%) possuíam cefaleia. Dos 46 portadores de cefaleia, 2 indivíduos (4,3\%) têm até 19 anos, 6 (13\%) têm entre 20 e 29 anos, 7 (15,2\%) têm entre 30 e 39 anos, 11 (23,9\%) estão na faixa dos 40 aos 49, 13 (28,2\%) na faixa dos 50 aos 59 anos e 7 (15,2\%) têm mais de 60 anos de idade.

\section{DISCUSSÃO}

O objetivo inicial deste estudo foi verificar a prevalência de cefaleia em indivíduos que frequentam um espaço ambulatorial. Os resultados mostraram que dos 87 entrevistados, 46 afirmaram ter cefaleia em suas diversas formas. Quando se analisam os dados referentes a cefaleia tensional (CT), percebe-se que a proporção de mulheres para homem é superior a 4:1, dado que corrobora um estudo de Bernardi (2008) sobre a referida cefaleia que afirma que a prevalência de cefaleia tensional é maior entre as mulheres.

Embora a CT seja amplamente apontada como mais prevalente das cefaleias, inclusive pela International Association for Study of Pain (IASP) em uma publicação de 2011, existem também autores que classificam como mais prevalente a migrânea, como é apontado no Global Burden of Diasease Survey de 2010.

Este, corroborado pelo presente estudo, onde a MCA foi responsável por $41 \%$ das queixas de cefaleia, seguida pela MSA representada por $32 \%$ dos relatos e por fim seguidas da CT ocupando apenas $23 \%$ das queixas. A diferença nos resultados das duas pesquisas mostra quão limitada ainda é a literatura sobre cefaleia e a importância de novas pesquisas para que se possa entender melhor as particularidades dessa manifestação tão relevante a fim de gerar ações mais eficientes no combate e prevenção das crises.

0 tabagismo também tem importante convergência com a cefaleia, alguns estudos tentaram esclarecer melhor essa relação, como o caso de Aamodt et al. (2006) em estudo que aponta maior prevalência de cefaleia entre fumantes que não fumantes englobando inclusive fumantes passivos. 0 que pode ser visto neste estudo, onde $24 \%$ dos entrevistados são fumantes, sendo que dentre os fumantes $47 \%$ sofrem de cefaleia. 0 tabaco além de desencadear um processo alérgico nas mucosas nasais, também possui, devido a nicotina, princípios vasoconstrictores elevando a pressão arterial e frequência cardíaca que podem influenciar na intensidade e incidência de crises de cefaleia (FRANKEN, 1995; ILIADES, 2011).

Quanto ao consumo alcoólico, dentre os entrevistados que referiram cefaleia, 16 indivíduos (34,7\%) alegaram etilismo. A fisiopatologia da relação entre álcool e cefaleia ainda não está completamente elucidada, é conhecido que o abuso de álcool induz às crises álgicas, porém, ainda não é possível afirmar que determinadas doses induzam ou não o surgimento de cefaleia (DUELAND, 2015).

Para alguns indivíduos, a prática de atividades físicas regulares pode ser um considerável fator de proteção, como descrito em alguns estudos pessoas que se exercitam regularmente de forma programada apresentam menor prevalência de cefaleia, além da prática regular de exercícios físicos interferir beneficamente na incidência de crises de cefaleia em determinados portadores mesmo embora a migrânea em alguns seja agravada ou desencadeada pela atividade física (SANTANA NETO, 2016) (KRYMCHANTOWSKI, 2006).

Nossa pesquisa revelou que $60,8 \%$ dos entrevistados com cefaleia são sedentários, o que afirma a maior prevalência de não realização de atividades físicas entre os acometidos pela patologia em questão.

Neste presente estudo quase metade $(43,4 \%)$ dos que referiram cefaleia não chegaram ao ensino médio, um parcela considerável e que tem relação descrita por SILVA JUNIOR et al, (2012), no estudo que aponta pessoas com baixa escolaridade mais propensas aos efeitos da cefaleia.

\section{CONCLUSÕES}

Considerando os pontos abordados na discussão, notase que muito pode ser feito ainda na atenção básica para melhorar a qualidade de vida de pessoas que sofrem com cefaleias recorrentes. É nítido o desconhecimento da população que depende do Sistema Único de Saúde (SUS) quanto a compreensão do seu próprio processo saúde-doença e o reflexo disso é uma população composta por desinformação que tenta buscar soluções caseiras e ineficientes ao invés de buscar auxílio dos profissionais de saúde. 
Em um segundo momento, outro objetivo deste estudo consiste em apresentar uma proposta de ação em saúde. Tendo em vista que, o SUS no Brasil vem adotando na Atenção Básica à Saúde (ABS) medidas públicas que visam implementar ações intersetoriais e multidisciplinares com a intenção de uma melhor promoção da saúde e prevenção de danos. Logo, notase um papel relevante da máquina pública em analisar estudos, projetar ações e executar medidas que colaborem para esse processo fundamental no que tange a saúde coletiva.

Além disso, ciente de que a promoção da saúde acontece quando uma população se apropria de conhecimentos e executa práticas necessárias para uma melhora da qualidade de vida e da saúde em geral, é necessário aplicar medidas que poderão auxiliar a população alvo do estudo no controle de suas crises e no processo de cronificação dessas patologias presentes no estudo. Visto que, a tarefa de promover saúde engloba vários fatores como aspectos comportamentais, estilo de vida, condições sociais e ambientais em que os indivíduos estão inseridos, logo, é de extrema importância desenvolver medidas que se aplicam nestes elementos.

Portanto, segundo Pinto (2019), além do tratamento medicamentoso para dor, existem medidas gerais que devem ser consideradas na profilaxia de novas crises ou na manutenção de sintomas. Em torno disso, reconhecer os fatores desencadeantes, sejam dietéticos por alimentação inadequada e ingredientes específicos ou por hábitos como uso e abuso de substâncias (tabaco, álcool, analgésicos, ansiolíticos, contraceptivo hormonal, entre outros), ritmo de sono, stress, situações emocionais, prática de atividades físicas, compreendem recomendações indispensáveis para um bom prognóstico.

Nesse sentido, para que a população tenha acesso a essa assistência adequada, será necessária uma maior disponibilização de equipes multiprofissionais composta por Médicos, Nutricionistas, Psicólogos e Educadores Físicos nas Unidades Básicas de Saúde da Família - (UBSF) e nas outras instituições que englobam as Estratégia Saúde da Família - (ESF). Sendo que, esses profissionais estejam aptos a desenvolverem ações de promoção da saúde que visam a adequada ação de hábitos de vida saudáveis e oferta de ações educativas, incentivando uma alimentação adequada e balanceada, bem como a prática de atividades físicas e mentais regularmente e ao abandono de vícios e comportamentos estressantes.

Além desses profissionais, outra possibilidade conveniente, será a implementação de estágios regulados por essa Instituição de Ensino em parceria com os gestores de saúde do município de Araguari-MG, de forma que acadêmicos dos cursos de Medicina, Nutrição, Educação Física e Psicologia poderão contribuir para a execução dessas ações em saúde de maneira benéfica à construção do conhecimento acadêmico e promoção de saúde à esta população estudada. Tendo em vista que, são capazes de realizar ações coletivas em que sejam promovidas medidas que visam orientar a população, expor conhecimentos e realizar acolhimentos dos usuários do sistema público de saúde.

\section{REFERÊNCIAS}

AAMODT, A. H., et al., Headache prevalence related to smoking and alcohol use. The Head-HUNT Study. European Journal of Neurology. Trondheim, Noruega, 2006.

\section{ASSOCIAÇÃO MÉDICA BRASILEIRA E CONSELHO FEDERAL DE MEDICINA. Cefaleias em Adultos na Atenção Primária à Saúde: Diagnóstico e Tratamento. Projeto diretrizes 2009.}

BERNARDI, M. T. et al. Correlação entre estresse e cefaleia tensional. Fisioterapia em Movimento, [S.L.], v. 21, п. 1, set. 2008.

BRASIL. Ministério Da Saúde. Secretaria de Políticas de Saúde. Projeto promoção da saúde. As cartas de promoção da saúde [Internet]. Brasília: Ministério da Saúde; 2002 [citado 2018 mar 6]. 56 p. (Série B. Textos Básicos em Saúde). Disponível em: Disponível em: http://bvsms.saude.gov.br/bvs/publicacoes/cartas_pro mocao.pdf. Acesso em: 29/11/2019

DITTRICH, S.M., et al. Aerobic exercise with relaxation: influence on pain and psychological well-being in female migraine patients. Clin J Sport Med. Innsbruck, Austria, 2008.

DUELAND, A. N. Headache and Alcohol. Headache: The Journal of Head and Face Pain, 2015.

FRANKEN, R. A. et al. Nicotina. Ações e interações. Arquivos Brasileiros de Cardiologia, São Paulo, volume 66, (n²), 1996.

HAMMILL, J.M., COOK, T.M., ROSECRANCE, J.C.: Effectiveness of a physical therapy regimen in the treatment of tension-type headache. Headache. lowa, Estados Unidos da América, 1996.

\section{ILIADES, C. 11 Biggest Headache}

Triggers. Disponivel em https://www.everydayhealth.com/headachemigraine/11-biggest-headache-triggers.aspx. Acesso em: 26/11/2019. 
KRYMCHANTOWSKI, A. V. et al, Prevalência e características da cefaleia em uma população de praticantes regulares de exercícios físicos. Arquivos de Neuropsiquiatria, vol.64 no.3b São Paulo Sept. 2006.

MEDINA M.G., et al. Promoção da saúde e prevenção de doenças crônicas: o que fazem as equipes de Saúde da Família? Saúde Debate. Rio de Janeiro, 2014, vol.38, n.spe, pp.69-82.

NARIN, S. et al. The effects of exercise and exerciserelated changes in blood nitric oxide level on migraine headache. Clinical rehabilitation. Turquia, 2003. Disponível em: https://journals.sagepub.com/doi/abs/10.1191/02692 15503cr657oa, acesso em 28/11/2019.

OLIVEIRA, M.A.C., PEREIRA, I.C.: Atributos essenciais da Atenção Primária e a Estratégia Saúde da Família. Revista Brasileira de Enfermagem. São Paulo, 2013;66(esp):158-64.

PORTO, C. C.; PORTO, A. L. Clínica Médica na prática diária. 1. ed. Rio de Janeiro: Guanabara Koogan, 2016.

SANTANA NETO, F. J. Efeitos de diferentes intensidades do exercício físico na cefaleia: uma revisão sistemática. 2016.

SILVA JUNIOR, A.A da. et al. Frequência dos tipos de cefaleia no centro de atendimento terciário do Hospital das Clínicas da Universidade Federal de Minas Gerais. Revista Associação Médica Brasileira. 58(6):709-713. Belo Horizonte, 2012.

SOCIEDADE INTERNACIONAL DE CEFALEIAS. Classificação Internacional de Cefaleias. 3. ed. Lisboa: Sociedade Internacional de Cefaleias, 2014. 